January 2017

\title{
Acute necrotizing encephalopathy of childhood secondary to dengue infection: A case report from Pakistan
}

Qalab Abbas

Aga Khan University, qalab.abbas@aku.edu

Sidra Kaleem Jafri

Aga Khan University

Sidra Ishaque

Aga Khan University, sidra.ishaque@aku.edu

Muhammad T.Jamil

Aga Khan University

Follow this and additional works at: https://ecommons.aku.edu/

pakistan_fhs_mc_women_childhealth_paediatr

Part of the Pediatrics Commons

\section{Recommended Citation}

Abbas, Q. Jafri, S. K., Ishaque, S., Jamil, M. T. (2017). Acute necrotizing encephalopathy of childhood secondary to dengue infection: A case report from Pakistan. Journal of Pediatric Neurosciences, 12(2), 165-167.

Available at: https://ecommons.aku.edu/pakistan_fhs_mc_women_childhealth_paediatr/403 


\title{
Case Report
}

\section{Acute Necrotizing Encephalopathy of Childhood Secondary to Dengue Infection: A Case Report from Pakistan}

\author{
Qalab Abbas, Sidra Kaleem Jafri, Sidra Ishaque, Muhammad T. Jamil
}

Department of Pediatrics and Child Health, Aga Khan University, Karachi, Pakistan

\begin{abstract}
Acute necrotizing encephalopathy of childhood (ANEC) is a rare condition mainly affecting children with a distinct clinico-radiologic pattern. Initially thought to be secondary to respiratory viral infections, there have been more insights to the pathogenesis of ANEC including genetics. We present a case of a girl who developed this condition with classical clinico-radiologic findings of ANEC secondary to severe dengue infection and could not survive. We report this case with the aim to raise awareness about this fatal complication of dengue infection as dengue has become a global health-care problem.
\end{abstract}

KEYWORDS: Acute necrotizing encephalopathy of childhood, children, dengue

\section{INTRODUCTION}

$\mathcal{D}$ engue is the second most common mosquito-borne illness affecting humans. ${ }^{[1]}$ It affects multiple organ systems of the body, including the central nervous system (CNS). Although rare, CNS manifestations or complications have been increasingly described in literature, especially during epidemics. ${ }^{[2,3]}$ These complications include dengue encephalopathy, encephalitis, immune complex-mediated syndromes, and dengue muscle dysfunction. ${ }^{[2,4]}$ While acute necrotizing encephalopathy of childhood (ANEC) was first described by Mizuguchi et al. in 1995, after that, multiple reports on ANEC have been published. ${ }^{[5,6]}$ ANEC usually affects younger children and has classical radiological findings of multifocal symmetrical lesions in thalami, brainstem, and cerebellum. ${ }^{[7]}$ Most of the cases of ANEC described in literature are reported to be secondary to different viral etiologies, especially respiratory viruses. This is the first case of ANEC secondary to dengue infection in Pakistan with classical clinicoradiological findings.

\section{CASe Report}

A 15-year-old, previously healthy school going girl presented to pediatric emergency department with a history of fever for 5 days and altered sensorium for 2 days. There was no associated cough, flu or breathing difficulty in her or any of the family member. Fever was high grade (up to103F), intermittent, with no aggravating

\begin{tabular}{|l|l|}
\hline \multicolumn{2}{|c|}{ Access this article online } \\
\hline Quick Response Code: & Website: \\
& \\
\hline
\end{tabular}

factors and relieved on taking antipyretics. Fever was associated with generalized continuous headache that was throbbing in nature. On the $4^{\text {th }}$ day of fever, she became dull and lethargic and developed vomiting for which she was taken to nearby physician where she was rehydrated. While being rehydrated, she had an episode of generalized, tonic-clonic seizure, lasting $15 \mathrm{~min}$ controlled with intravenous diazepam. Her consciousness further deteriorated, and she was brought to our hospital. On examination, she was tachycardiac and hypotensive with Glasgow coma scale (GCS) of 6/15 (E2V1M3). Her pupils were constricted bilaterally with intact cough and gag reflex and no facial weakness. She had generalized increased tone, brisk reflexes, and up going planters. Signs of meningeal irritation were positive. There was no facial dysmorphism, visceromegaly, or any rash. Initial impression of meningoencephalitis and space occupying lesion was made. Her laboratory workup showed hemoglobin $12.4 \mathrm{~g} / \mathrm{dl}$ (hematocrit 36.5\%), total leukocytes count 10500 with $85 \%$ neutrophils, and thrombocytopenia (platelets 70,000). Her transaminases were elevated with reversal of alanine transaminase (ALT) to aspartate transaminase (AST)

\section{Address for correspondence: Dr. Qalab Abbas, Department of Pediatrics and Child Health, Aga Khan University, Karachi, Pakistan. E-mail: qalababbas@gmail.com}

This is an open access article distributed under the terms of the Creative Commons Attribution-NonCommercial-ShareAlike 3.0 License, which allows others to remix, tweak, and build upon the work non-commercially, as long as the author is credited and the new creations are licensed under the identical terms.

For reprints contact: reprints@medknow.com

How to cite this article: Abbas Q, Jafri SK, Ishaque S, Jamil MT. Acute necrotizing encephalopathy of childhood secondary to dengue infection: A case report from Pakistan. J Pediatr Neurosci 2017;12:165-7. 
ratio (ALT $507 \mathrm{IU} / \mathrm{L}$, AST $1165 \mathrm{IU} / \mathrm{L}$ ). Her creatinine and electrolytes were normal. Plain computed tomography $(\mathrm{CT})$ of the brain was initially done which showed diffuse low-attenuation area in the pons which was extending into the right basal ganglia with slight expansion of the pons. This was followed by magnetic resonance imaging (MRI) brain which showed abnormal, symmetrical signals in the thalamus, midbrain, pons, and white matter of the cerebellar hemispheres. These lesions were appearing hyperintense on T2-weighted images, hypointense on T1-weighted images, and showing minimal postcontrast enhancement. Necrotic areas were also identified in the right thalamus and both sides of the pons showing peripheral enhancement and diffusion restriction [Figure 1]. The findings were suggestive of ANEC. Nasopharyngeal swab for influenza A, B, H1N1 and other respiratory viruses, cerebrospinal fluid for herpes simplex virus polymerase chain reaction (PCR) were negative. Her dengue immunoglobulin $\mathrm{M}(\operatorname{IgM})$ sent due to high-grade fever, and thrombocytopenia came out to be positive. She was electively intubated and mechanically ventilated, and she was provided neuroprotective mesures along with pulse dose methylprednisolone and intravenous immunoglobulin. She was also started on oseltamivir and acyclovir. After $24 \mathrm{~h}$, she went into multiorgan failure (MOF) requiring high inotropic and ventilatory support. She continued to deteriorate and her GCS decreased to $2 / 10$ and her cough and gag were lost. On the $3^{\text {rd }}$ day of admission, she fulfilled brain death criteria and expired.

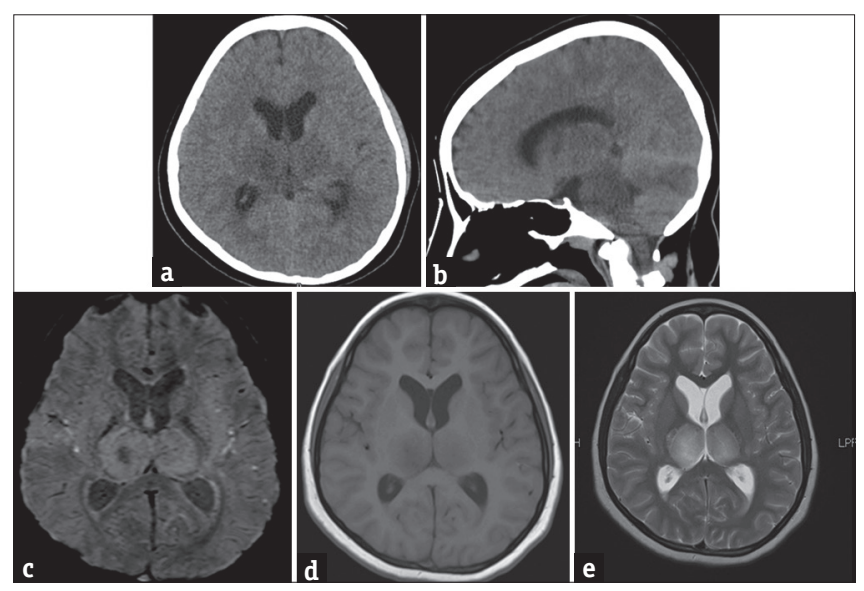

Figure 1: Computed tomography and magnetic resonance imaging of the patient. (a) Computed tomography scan brain showing diffuse low attenuation areas in pons with effacement of the lateral ventricle. (b) Computed tomography scan brain showing diffuse low attenuation areas in pons with gradual transition to midbrain and medulla. (c) Abnormal, symmetrical signals in the thalamus and midbrain. (d) Lesions are appearing hypointense on T1-weighted images. (e) Hyperintense on T2-weighted images and showing minimal postcontrast enhancement. Necrotic areas are also identified in the right thalamus and both sides of the pons which are showing peripheral enhancement

\section{Discussion}

This child had presented with acute onset fever and drowsiness and was initially suspected of having a CNS infection. She had signs of raised intracranial pressure, and a CT of the brain was done before attempting a lumbar puncture. CT showed a diffuse attenuation of pons extending to basal ganglia, so we proceeded with an MRI which showed classical radiological findings of ANEC. Thrombocytopenia with raised liver enzymes levels prompted us to send the dengue antibodies as dengue infection is prevalent in our part of the world, which came positive while nasal swab for reverse transcriptase PCR of influenza A, B, and H1N1 was negative. We observed the typical course of rapid progression of neurological deterioration of ANEC in our patient. ${ }^{[6]}$ However, the development of MOF has not been described in ANEC patients earlier which could be due to severe dengue infection. The exact etiology of ANEC has yet to be determined, but ANEC has been linked to certain respiratory infections such as influenza and genetics have also been described to be playing a role. ${ }^{[8]}$ The most commonly accepted hypothesis regarding it pathogenesis is hypercytokinemia. ${ }^{[7]}$ This hypercytokinemia ("cytokine storm") can eventually lead to multiorgan dysfunction as observed in our patient.

Although CNS involvement with dengue infection has been well described the types of brain lesions (typical of ANEC), we observed in our patient have not been described before in literature. ${ }^{[2,4]}$ As dengue has been spreading to more parts of the world more new complications have been observed. In addition, the pathology of such lesion of ANEC in our patient secondary to dengue could possibly be same as has been described in other viral etiologies.

Management of ANEC is mainly supportive with antiepileptic drugs and neuroprotective measures. Some studies have shown the beneficial role of antiviral agents such as amantadine and oseltamivir along with methylprednisolone pulse doses and intravenous Ig. ${ }^{[9]}$ The outlook of ANEC is very poor with $30 \%$ patients dying during the acute illness and those who survive have a significant neurological impairment. ${ }^{[6]}$ Our patient had brainstem involvement, and it leads to brain death while a report from Korea showed that $40 \%$ of their patient with brainstem involvement expired during the acute illness and another 30\% developed severe neurological impairment. ${ }^{[10]}$

To the best of our knowledge, this is the first report of ANEC secondary to dengue infection with a very fulminant course. With the passage of time and more awareness, the outcome of ANEC is improving but it 
still remains a deadly disease. The availability of dengue vaccination could be the only way to prevent these deadly complications of dengue infection.

\section{Financial support and sponsorship}

Nil.

\section{Conflicts of interest}

There are no conflicts of interest.

\section{REFERENCES}

1. Bhatt S, Gething PW, Brady OJ, Messina JP, Farlow AW, Moyes CL, et al. The global distribution and burden of dengue. Nature 2013;496:504-7.

2. Carod-Artal FJ, Wichmann O, Farrar J, Gascón J. Neurological complications of dengue virus infection. Lancet Neurol 2013;12:906-19.

3. Madi D, Achappa B, Ramapuram JT, Chowta N, Laxman M, Mahalingam S. Dengue encephalitis - A rare manifestation of dengue fever. Asian Pac J Trop Biomed 2014;4 Suppl 1:S70-2.
4. Puccioni-Sohler M, Rosadas C, Cabral-Castro MJ. Neurological complications in dengue infection: A review for clinical practice. Arq Neuropsiquiatr 2013;71:667-71.

5. Khan MR, Maheshwari PK, Ali SA, Anwarul H. Acute necrotizing encephalopathy of childhood: A fatal complication of swine flu. J Coll Physicians Surg Pak 2011;21:119-20.

6. Mizuguchi M. Acute necrotizing encephalopathy of childhood: A novel form of acute encephalopathy prevalent in Japan and Taiwan. Brain Dev 1997;19:81-92.

7. Wu X, Wu W, Pan W, Wu L, Liu K, Zhang HL. Acute necrotizing encephalopathy: An underrecognized clinicoradiologic disorder. Mediators Inflamm 2015;2015:792578.

8. Neilson DE. The interplay of infection and genetics in acute necrotizing encephalopathy. Curr Opin Pediatr 2010;22:751-7.

9. Okumura A, Mizuguchi M, Kidokoro H, Tanaka M, Abe S, Hosoya $\mathrm{M}$, et al. Outcome of acute necrotizing encephalopathy in relation to treatment with corticosteroids and gammaglobulin. Brain Dev 2009;31:221-7.

10. Lee CG, Kim JH, Lee M, Lee J. Clinical outcome of acute necrotizing encephalopathy in related to involving the brain stem of single institution in Korea. Korean J Pediatr 2014;57:264-70. 